\title{
Classical predictive electrodynamics of two charges with radiation: Energy and 3-momentum balance and scattering cross sections. II
}

\author{
R. Lapiedra \\ Departamento de Física Teórica, Facultad de Ciencias, Santander, Spain \\ F. Marqués and A. Molina \\ Departamento de Fisica Teórica, Facultad de Física, Barcelona, Spain
}

(Received 7 July 1978; revised manuscript received 6 November 1978)

\begin{abstract}
We deal with a classical predictive mechanical system of two spinless charges where radiation is considered and there are no external fields. The terms ${ }^{(2.2)} P_{a}^{a}$ of the expansion in the charges of the Hamilton-Jacobi momenta are calculated. Using these, together with known previous results, we can obtain the $p_{a}^{\alpha}$ up to the fourth order. Then we have calculated the "radiated" energy and the 3-momentum in a scattering process as functions of the impact parameter and the incident energy for the former and 3-momentum for the latter. Scattering cross-sections are also calculated. Good agreement with well known results, including those of quantum electrodynamics, has been found.
\end{abstract}

\section{INTRODUCTION}

In this paper we pursue the calculations of the preceding paper (I) (this issue) in order to obtain physical results such as the cross sections and the "radiated" energy and 3momentum. [We explain what is to be considered as radiated energy and 3-momentum in paper I (Sec. 5) and again at the end of Sec. 3 in this paper.] We use the notation and general scheme developed in Ref. 1, i.e., a classical predictive mechanical system consisting of two spinless charges, each one moving in the field of the other according to the appropriate Lorentz-Dirac equation.

Let us summarize the relevant results of (I): In a perturbative scheme in the charges, $e_{a}$, the 4-accelerations of the "auxiliary dynamical system" (I.4.12) are determined up to fourth order (included). [(I.4.12) means the formula (4.12) of Ref. 1.] The terms ${ }^{(1,1)} \theta_{a}^{\alpha}$ and ${ }^{(2,2)} \theta_{a}^{\alpha}$ of the expansion are the same as if radiation (that is, the Dirac term in the Lorentz-Dirac equation) was not present and they were already known. The term ${ }^{(1,1)} \theta_{a}^{\alpha}$ can be found in Ref. 1 . On the other hand, the terms ${ }^{(2,2)} \xi_{a}^{\alpha}$, of the original dynamical system's accelerations, can be found in Ref. 2. From them, the terms ${ }^{(2,2)} \theta_{a}^{\alpha}$ are easily calculated. They are given in Appen$\operatorname{dix}$ B. The terms ${ }^{(3,1)} \theta_{a}^{\alpha}$, calculated in (I), are new. The terms ${ }^{(1,3)} \theta_{a}^{\alpha}$ can be seen to be absent.

The Hamilton-Jacobi coordinates $\tilde{p}_{a}^{\alpha}, \tilde{q}_{a}^{\alpha}$, can be computed taking into account the 4-accelerations, $\theta_{a}^{\alpha}$, of "the auxiliary dynamical system." The first terms ${ }^{(1,1)} \widetilde{p}_{a}^{\alpha},{ }^{(1,1)} \tilde{q}_{a}^{\alpha}$, in the expansions of $\tilde{p}_{a}^{\alpha}, \tilde{q}_{a}^{\alpha}$ are obtained in Ref. 1 . In (I) we have obtained the terms ${ }^{(3.1)} \tilde{p}_{a}^{\alpha},{ }^{(3.1)} \tilde{q}_{a}^{\alpha}$ (the terms ${ }^{(1,3)} \tilde{p}_{a}^{\alpha},{ }^{(1.3)} \tilde{q}_{a}^{\alpha}$ can be taken equal to zero). In order to have all terms to fourth order in $\tilde{p}_{a}^{\alpha}, \tilde{q}_{a}^{\alpha}$, the terms ${ }^{(2,2)} \tilde{p}_{a}^{\alpha},{ }^{(2,2)} \tilde{q}_{a}^{\alpha}$ should also be calculated. Their calculation follows the same lines as that of ${ }^{(3,1)} \widetilde{p_{a}^{\alpha}},{ }^{(3,1)} \tilde{q}_{a}^{\alpha}$, but it is very cumbersome. Nevertheless if any more concrete results are to be obtained from our mechanical system, knowledge of ${ }^{(2.2)} p_{a}^{\alpha}$ is needed.

In Sec. 1 we will compute ${ }^{(2.2)} p_{a}^{\alpha}$ and so we will get the total 4-momentum, $P^{\alpha}=p_{1}^{\alpha}+p_{2}^{\alpha}$, of the system to fourth order. In Sec. 2 the future (respectively past) infinite limit of the calculated Hamilton-Jacobi momenta, $p_{a}^{\alpha}$, are calculated. In this way we can establish the 4-momentum balance of the interaction and so, in our approximation. We see that there is no energy and 3-momentum "radiated". In Sec. 3 we use the asymptotic behavior of $p_{a}^{\alpha}$ in order to calculate the scattering cross section of the classical process, to sixth order in $e_{1}, e_{2}$. In the limit where sixth order terms are negligible, our results agree with the previous one of $\mathrm{Bel}^{3}$ and they are also shown to agree with those of quantum electrodynamics.

\section{THE CALCULATION OF THE TERMS ${ }^{(2,2)} p_{a}^{\alpha}$ IN THE HAMILTON-JACOBI MOMENTA EXPANSION}

We start with the first equation in (I.4.6), written for the "auxiliary dynamical system" (I.4.12),

$$
\frac{d \tilde{p}_{a}^{\alpha}}{d s_{b}}=0,
$$

where

$$
\frac{d}{d s_{b}}=\pi_{b}^{\rho} \frac{\partial}{\partial x^{b \rho}}+\theta_{b}^{\rho} \frac{\partial}{\partial \pi^{b \rho}} .
$$

Fourth order terms of the form (2.2) (terms in $e_{a}^{2} e_{a^{\prime}}^{2}$ ) in (1.1) give

$$
\pi_{b}^{\rho} \frac{\partial^{(2,2)} \tilde{p}_{a}^{\alpha}}{\partial x^{b \rho}}=-{ }^{(1,1)} \theta_{b}^{\rho} \frac{\partial^{(1,1)} \tilde{p}_{a}^{\alpha}}{\partial \pi^{b \rho}}-{ }^{(2,2)} \theta_{b}^{\rho} \frac{\partial^{(0,0)} \tilde{p}_{a}^{\alpha}}{\partial \pi^{b \rho}}
$$

or taking into account (I.4.18) and (I.5.6),

$$
D_{b}{ }^{(2,2)} \tilde{p}_{a}^{\alpha}=-{ }^{(1,1)} \theta_{b}^{\rho} \frac{\partial^{(1,1)} \tilde{p}_{a}^{\alpha}}{\partial \pi^{b \rho}}-{ }^{(2,2)} \theta_{a}^{\alpha} \delta_{a b},
$$

that is,

$$
D_{a}{ }^{(2,2)} \tilde{p}_{a}^{\alpha}=-{ }^{(1,1)} \theta_{a}^{\rho} \frac{\partial^{(1,1)} \tilde{p}_{a}^{\alpha}}{\partial \pi^{a \rho}}-{ }^{(2,2)} \theta_{a}^{\alpha}
$$

and 


$$
D_{a^{\prime}}{ }^{(2,2)} \tilde{p}_{a}^{\alpha}=-{ }^{(1,1)} \theta_{a^{\prime}}^{\rho} \frac{\partial^{(1,1)} \tilde{p}_{a}^{\alpha}}{\partial \pi^{a^{\prime} \rho}} .
$$

Now, for ${ }^{(1.1)} \theta_{a}^{\rho}$ we get [see (1.3.7) and (1.4.13)]

$$
{ }^{(1,1)} \theta_{a}^{\rho}=\frac{\eta_{a} \pi_{a}^{2} \widetilde{k}}{\tilde{r}_{a}^{3}} \widetilde{h}^{\rho}-\frac{\pi_{a^{2}}^{2} \tilde{z}_{a}}{\tilde{r}_{a}^{3}} \widetilde{t}_{a}^{\rho},
$$

and from Ref. 1 one has for ${ }^{(1,1)} \tilde{p}_{a}^{\alpha}$

$$
{ }^{(1.1)} \tilde{p}_{a}^{\alpha}=\frac{\eta_{a} \widetilde{k}}{\widetilde{h}^{2}}\left(\frac{\gamma}{\widetilde{\Lambda}}-\frac{\tilde{z}_{a}}{\tilde{r}_{a}}\right) \widetilde{h}^{\alpha}-\frac{\pi_{a^{\prime}}^{2}}{{\widetilde{\Lambda^{2}}}^{2} \tilde{r}_{a}^{\alpha}}{ }_{a^{\prime}},
$$

where $\gamma$ stands for +1 or -1 , according to whether we want ${ }^{(1,1)} \tilde{p}_{a}^{\alpha}$ to be zero at the future infinite or at the past infinite. (When comparing with Ref. 1 attention must be paid to the fact that there is a slight change of notation for the symbol $\tilde{r}_{a}$.) It can readily be verified from (1.8) that

$$
\lim _{z_{, \prime \prime \rightarrow \gamma^{\prime} \infty}}\left[\frac{\eta_{a} \tilde{k}}{\widetilde{h^{2}}}\left(\frac{\gamma}{\widetilde{\Lambda}}-\frac{\tilde{z}_{a}}{\tilde{r}_{a}}\right) \widetilde{h}^{\alpha}-\frac{\pi_{a^{\prime}}^{2}}{\widetilde{\Lambda}^{2} \tilde{r}_{a}}{\widetilde{a^{\prime}}}^{\alpha}\right]=0 .
$$

Now, as we know, the symbol $\sim$ in the notation $\widetilde{k}, \widetilde{h}$, $\widetilde{A}, \ldots$, etc., means that we refer to "the auxiliary dynamical system" (I.4.12) and then we have the definitions (I.4.14), (I.4.15), $\cdots$, while $k, h, A, \cdots$ refer to the definitions (I.3.3),

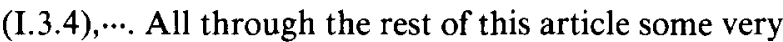
lengthy expressions will appear, we will omit from now on the symbol $\sim$ when refering to "the auxiliary dynamical system." So we will write $h^{\alpha}, t_{a}^{\alpha}, z_{a}, \cdots$, for $\widetilde{h}^{\alpha}, \widetilde{t}_{a}^{\alpha}, \tilde{z}_{a}, \cdots$. Later on we will have to work with the original dynamical system. Then, symbols without a tilde will again have their original meaning. This will be pointed out when necessary.

Now let us come to Eq. (1.5) and (1.6), which we must integrate in order to find ${ }^{(2,2)} \tilde{p}_{a}^{\alpha}$. For this we first need to compute ${ }^{(1,1)} \theta_{b}^{\rho}\left(\partial^{(1,1)} \tilde{p}_{a}^{\alpha} / \partial \pi^{b \rho}\right)$. Taking into account (1.7) and (1.8) we find after some lengthy calculations (see Appendix A for details)

$$
\begin{aligned}
& { }^{(1,1)} \theta_{a}^{\rho} \frac{\partial^{(1.1)} \tilde{p}_{a}^{\alpha}}{\partial \pi^{a \rho}}=\frac{\eta_{a} \pi_{a}^{2} \Lambda^{2} z_{a}}{h^{2} r_{a}^{3}}\left(\frac{\gamma}{\Lambda}-\frac{z_{a}}{r_{a}}\right) \\
& +\frac{\pi_{a^{\prime}}^{2}}{r_{a}^{3}}\left(\frac{z_{a}}{r_{a}}-\frac{\gamma k^{2}}{\Lambda^{3}}\right) t_{a}^{\alpha} \\
& { }^{(1,1)} \theta_{a}^{\rho} \frac{\partial^{(1,1)} \tilde{p}_{a}^{\alpha}}{\partial \pi^{a \rho}} \\
& =\frac{\eta_{a} \pi_{a}^{2}}{r_{a^{\prime}}^{3}}\left(\frac{k z_{a}}{h^{2} r_{a}^{3}}\left(k z_{a}-\pi_{a^{2}}^{2} z_{a^{\prime}}\right)\left(k h^{2}+\Lambda^{2} z_{a^{\prime}} z_{a^{\prime}}\right)\right. \\
& -\frac{1}{h^{2} r_{a}}\left[k z_{a^{\prime}}\left(k z_{a}-\pi_{a^{2}}^{2} z_{a^{\prime}}\right)+\left(k h^{2}+\Lambda^{2} z_{a^{2}} z_{a^{\prime}}\right)\right] \\
& \left.+\frac{\gamma \Lambda z_{a^{\prime}}}{h^{2}}\right) h^{\alpha}+\frac{\pi_{a}^{2}}{r_{a}^{3}}\left(\frac{\pi_{a^{\prime}}^{2}}{\Lambda^{2} r_{a}^{3}}\left(k z_{a}-\pi_{a^{\prime}}^{2} z_{a^{\prime}}\right)\left(k h^{2}+\Lambda^{2} z_{a^{\prime}} z_{a^{\prime}}\right)\right. \\
& \left.-\frac{k}{\Lambda^{2} r_{a}}\left(k z_{a}-\pi_{a^{\prime}}^{2} z_{a^{\prime}}\right)+\frac{\gamma k^{2}}{\Lambda^{3}}\right) t_{a^{\prime}}^{\alpha},
\end{aligned}
$$

that is, ${ }^{(1,1)} \theta_{b}^{\rho}\left(\partial^{(1,1)} \tilde{p}_{a}^{\alpha} / \partial \pi^{b \rho}\right)$ can be written in the form

$$
{ }^{(1,1)} \theta_{b}^{\rho} \frac{\partial^{(1,1)} \tilde{p}_{a}^{\alpha}}{\partial \pi^{b \rho}}=\eta_{a}^{(2,2)} b_{b} h^{\alpha}+{ }^{(2,2)} C_{b} t_{b}^{\alpha},
$$

where the functions ${ }^{(2,2)} b_{b}$ and ${ }^{(2,2)} C_{b}$ are those appearing in (1.10) and (1.11).

On the other hand, the terms ${ }^{(2.2)} \xi_{a}^{\alpha}$ in the accelerations $\xi_{a}^{\alpha}$ of our predictive dynamical system can be found in Ref. 4 or, in a more compact writing, in Ref. 2 . from ${ }^{(2,2)} \xi_{a}^{\alpha}$ the term ${ }^{(2,2)} \theta_{a}^{\alpha}$ can be calculated according to (I.4.13). It can be found in Appendix B. Its expression has the form

$$
{ }^{(2,2)} \theta_{a}^{\alpha}=\eta_{a}{ }^{(2,2)} a_{a} h^{\alpha}+{ }^{(2,2)} l_{a a^{\prime}} t_{a^{\prime}}^{\alpha},
$$

with ${ }^{(2,2)} a_{a}{ }^{(2,2)} l_{a a^{\prime}}$ being the appropriate functions.

Finally, according to the notation of (I.5.3) we write

$$
{ }^{(2,2)} \tilde{p}_{a}^{\alpha}=\eta_{a}{ }^{(2,2)} \widetilde{\alpha}_{a} h^{\alpha}+{ }^{(2,2)} \tilde{\mu}_{a a} t_{a}^{\alpha}+{ }^{(2,2)} \widetilde{\mu}_{a a} t_{a}^{\alpha} \text {. }
$$

Now, putting (1.12), (1.13), (1.14), into (1.5), (1.6) and keeping in mind (I.5.7), we have

$$
\begin{aligned}
D_{a}^{(2,2)} \widetilde{\alpha}_{a} & =-{ }^{(2,2)} b_{a}-{ }^{(2,2)} a_{a}, D_{a}{ }^{(2,2)} \widetilde{\mu}_{a a} \\
& =-{ }^{(2,2)} C_{a}, \quad D_{a}{ }^{(2,2)} \widetilde{\mu}_{a a^{\prime}}=-{ }^{(2,2)} l_{a a^{\prime}} \\
D_{a^{\prime}}{ }^{(2,2)} \widetilde{\alpha}_{a} & =-{ }^{(2,2)} b_{a^{\prime}}, \quad D_{a^{\prime}}{ }^{(2,2)} \widetilde{\mu}_{a a}=0 \\
D_{a^{\prime}}{ }^{(2,2)} \widetilde{\mu}_{a a^{\prime}} & =-{ }^{(2,2)} C_{a^{\prime}} .
\end{aligned}
$$

Here, Eqs. (1.15) are equivalent to (1.5), and equations (1.16) to $(1.6)$

Because of (1.5.11), taking $\pi_{a}^{2}, h^{2}, \Lambda^{2}, z_{a}$ as independent variables, $\mathrm{E}$ qs. (1.15) and (1.16) can also be written

$$
\begin{aligned}
\frac{\partial^{(2,2)} \alpha_{a}}{\partial z_{a}} & =-{ }^{(2,2)} b_{a}-{ }^{(2,2)} a_{a}, \quad \frac{\partial^{(2,2)} \mu_{a a}}{\partial z_{a}} \\
& =-{ }^{(2,2)} C_{a}, \quad \frac{\partial^{(2,2)} \mu_{a a^{\prime}}}{\partial z_{a}}=-{ }^{(2,2)} l_{a a^{\prime}},
\end{aligned}
$$

$$
\begin{aligned}
& \frac{\partial^{(2,2)} \alpha_{a}}{\partial z_{a^{\prime}}}=-{ }^{(2,2)} b_{a^{\prime}}, \quad \frac{\partial^{(2,2)} \mu_{a a}}{\partial z_{a^{\prime}}}=0, \\
& \frac{\partial^{(2,2)} \mu_{a a^{\prime}}}{\partial z_{a^{\prime}}}=-{ }^{(2,2)} C_{\alpha^{\prime}}
\end{aligned}
$$

We will use these equations to determine ${ }^{(2,2)} \alpha_{a}$ and ${ }^{(2,2)} \mu_{a a}$, while ${ }^{(2,2)} \mu_{a a}$ will be obtained from (I.4.19). Let us begin with ${ }^{(2,2)} \alpha_{a}$. We will have

${ }^{(2,2)} \widetilde{\alpha}_{a}=\int_{z_{u}}^{\gamma \infty}\left({ }^{(2,2)} b_{a}+{ }^{(2,2)} a_{a}\right) d z_{a}+\int_{z_{, \prime}}^{\gamma \propto}\left(\lim _{z_{a} \rightarrow \gamma_{\infty}}{ }^{(2,2)} b_{a^{\prime}}\right) d z_{a^{\prime}}$

and depending on whether we take $\gamma=+1$ or $\gamma=-1$ we will have $\lim ^{(2,2)} \alpha_{a}=0$ at the future or past infinite respectively. In a similar way we have 


$$
{ }^{(2,2)} \tilde{\mu}_{a a^{\prime}}=\int_{z_{u^{\prime}}}^{\gamma_{\infty}}{ }^{(2,2)} l_{a a^{\prime}} d z_{a}+\int_{z_{z^{\prime \prime}}}^{\gamma \infty}\left(\lim _{z_{u} \rightarrow \gamma_{\infty}}{ }^{(2,2)} C_{a^{\prime}}\right) d z_{a^{\prime}}
$$

Taking into account (1.12) and (1.11), it is easy to see that

$$
\lim _{z_{1, \cdots} \rightarrow \gamma_{\infty}^{\infty}}{ }^{(2,2)} b_{a^{\prime}}=0, \quad \lim _{z_{1, \cdots+\gamma \infty}}{ }^{(2,2)} C_{a^{\prime}}=0,
$$

so that (1.19) and (1.20) become:

$$
\begin{aligned}
& { }^{(2,2)} \tilde{\alpha}_{a}=\int_{z^{\prime \prime}}^{\gamma \infty}\left({ }^{(2,2)} b_{a}+{ }^{(2,2)} a_{a}\right) d z_{a}, \\
& { }^{(2,2)} \bar{\mu}_{a a^{\prime}}=\int_{z^{\prime}}^{\gamma_{\infty}}{ }^{(2,2)} l_{a a^{\prime}} d z_{a} .
\end{aligned}
$$

Now, as it can be seen in Appendix B, ${ }^{(2,2)} a_{a},{ }^{(2,2)} l_{a a}$, are rather involved expressions. Consequently these integrals, though expressible as elementary functions, are involved too. The final result is:

$$
\begin{aligned}
& { }^{(2,2)} \widetilde{\alpha}_{a} \\
& =\frac{\pi_{a}^{2} \pi_{a^{4} k}}{\left(k r_{a}-\Lambda^{2} z_{a}\right)\left(k^{2} r_{a}^{2}-\Lambda^{4} z_{a}^{2}\right)}+\frac{\pi_{a}^{2} \pi_{a^{\prime}}^{2}}{2 \Lambda^{2} h^{2}} \\
& \times \frac{\left(2 k^{2}-\Lambda^{2}\right) r_{a}+k \Lambda^{2} z_{a}}{k^{2} r_{a}^{2}-\Lambda^{4} z_{a}^{2}}+\frac{\pi_{a}^{2}\left(2 \pi_{a}^{2} k-\Lambda^{2}\right) z_{a}}{2 \Lambda^{2} h^{2} r_{a}^{2}} \\
& -\frac{\pi_{a}^{2}}{\Lambda^{2} h^{2} r_{a^{\prime}} r_{a}}\left(\pi_{a^{\prime}}^{2} r_{a^{\prime}}+\pi_{a}^{2} k z_{a^{\prime}}+\Lambda^{2} z_{a}\right)+\frac{k \pi_{a^{\prime}}^{2}}{\Lambda^{2} r_{a^{\prime}} r_{a}^{3}} \\
& \times\left[k r_{a^{\prime}}+\left(2 k^{2}-\Lambda^{2}\right) z_{a^{\prime}}-2 \pi_{a}^{2} k z_{a}\right]+\frac{\gamma}{\Lambda h^{2}} \\
& \times\left(\frac{\pi_{a}^{2}}{r_{a^{\prime}}}+\frac{\pi_{a^{\prime}}^{2}}{r_{a}}\right)-\frac{\pi_{a}^{2} \pi_{a^{4}}^{4} k}{\Lambda^{3} r_{a}^{3}} \log \frac{\left(r_{a}-\Lambda z_{a}\right)\left(r_{a^{\prime}}+\Lambda z_{a^{\prime}}\right)}{(k-\Lambda) h^{2}} \\
& +\frac{\pi_{a}^{2} \pi_{a}^{\prime} k}{\Lambda^{3} h^{3}} \arctan \frac{r_{a}-\Lambda z_{a}}{\pi_{a^{\prime}} h}+\frac{\pi_{a}^{\prime}\left(\pi_{a}^{2} k+\Lambda^{2}\right)}{2 \Lambda^{3} h^{3}} \\
& \times \arctan \frac{\Lambda z_{a}}{\pi_{a^{\prime}} h}+\frac{\pi_{a}}{2 \Lambda h^{3}}\left(\arctan \frac{\pi_{a_{a}} r_{a}}{\Lambda h}+\arctan \frac{\pi_{a} \Lambda z_{a}}{k h}\right) \\
& -\frac{\pi \pi_{a}^{2} \pi_{a^{\prime}} k}{4 \Lambda^{3} h^{3}}-\frac{\pi \pi_{a}}{4 \Lambda h^{3}}-\frac{\gamma \pi\left(\pi_{a}+\pi_{a^{\prime}}\right)}{4 \Lambda h^{3}}, \\
& { }^{(2,2)} \widetilde{\mu}_{a a^{\prime}} \\
& =\frac{\pi_{a}^{2} \pi_{a^{\prime}}^{2}}{2 \Lambda^{2} h^{2}} \frac{\pi_{a}^{2} h^{2}-2 k r_{a} z_{a}}{k^{2} r_{a}^{2}-\Lambda^{4} z_{a}^{2}}-\pi_{a^{\prime}}^{4} \frac{\pi_{a}^{2} k r_{a} z_{a}+k^{2} h^{2}}{\left(k^{2} r_{a}^{2}-\Lambda^{4} z_{a}^{2}\right)^{2}} \\
& +\frac{\pi_{a}^{2} \pi_{a^{\prime}}^{4}}{\Lambda^{4} r_{a}^{2}}+\frac{2 \pi_{a}^{2} \pi_{a^{2}}^{2} k}{\Lambda^{4} r_{a} r_{a^{\prime}}}-\frac{2 \pi_{a}^{2} \pi_{a^{4}}^{4} k h^{2}}{\Lambda^{4} r_{a}^{3} r_{a^{\prime}}}
\end{aligned}
$$

$$
\begin{aligned}
& +\frac{\left.k z_{a} k z_{a^{\prime}}+r_{a^{\prime}}\right)}{\Lambda^{2} h^{2} r_{a} r_{a^{\prime}}}-\frac{z_{a} \pi_{a^{\prime}}^{2}\left[k r_{a^{\prime}}+\left(2 k^{2}-\Lambda^{2}\right) z_{a^{\prime}}\right]}{\Lambda^{2} r_{a^{3}}^{3} r_{a^{\prime}}} \\
& -\gamma \frac{k^{2} z_{a^{\prime}}}{\Lambda^{3} h^{2} r_{a^{\prime}}}-\frac{\pi_{a}^{2} \pi_{a^{4}}^{4} z_{a}}{\Lambda^{3} r_{a}^{3}} \log \frac{\left(r_{a}+\Lambda z_{a}\right)(k-\Lambda)}{\pi_{a^{\prime}}^{2}\left(r_{a^{\prime}}+\Lambda z_{a^{\prime}}\right)} .
\end{aligned}
$$

Let us now calculate ${ }^{(2,2)} \mu_{a a^{\prime}}$. Keeping fourth order terms in (I.4.19) we obtain

$$
2^{(2,2)} \tilde{p}_{a}^{\alpha} \pi_{a \alpha}+{ }^{(1,1)} \tilde{p}_{a}^{\alpha(1,1)} \tilde{p}_{a \alpha}=0
$$

and from this equation and (I.5.3)

$$
{ }^{(2,2)} \widetilde{\mu}_{a a}=-\frac{h^{2(1,1)} \widetilde{\alpha}_{a}^{2}+\pi_{a}^{2} \Lambda^{2(1,1)} \widetilde{\mu}_{a \alpha}^{2}}{2 \Lambda^{2}} .
$$

Then, because of (1.8), we find

$$
{ }^{(2,2)} \tilde{\mu}_{a a}=\frac{\pi_{a^{\prime}}^{2}}{2 \Lambda^{2} r_{a}^{2}}+\frac{k^{2}}{\Lambda^{3} h^{2}}\left(\frac{\gamma z_{a}}{r_{a}}-\frac{1}{\Lambda}\right) .
$$

Finally, substitution of (1.28), (1.25), and (1.24) in (1.14) gives us ${ }^{(2,2)} \tilde{p}_{\alpha}^{\alpha}$. Then from (I.4.18) and (I.5.14) we can write

$$
\begin{aligned}
\tilde{p}_{a}^{\alpha}= & \pi_{a}^{\alpha}+e_{1} e_{2}\left[\frac{\eta_{a} k}{h^{2}}\left(\frac{\gamma}{k}-\frac{z_{a}}{r_{a}}\right) h^{\alpha}-\frac{\pi_{\alpha^{\prime}}^{2}}{\Lambda^{2} r_{a}} t_{a^{\prime}}^{\alpha}\right] \\
& +e_{a}^{3} e_{a^{\prime}}\left[-\frac{2 \eta_{a} \pi_{a^{2}}^{2} k}{3 \pi_{a}^{2} r_{a}^{3}} h^{\alpha}+\frac{2 \pi_{a^{2}}^{2} z_{A}}{3 \pi_{a}^{2} r_{a}^{3}} t_{a^{\prime}}^{\alpha}\right] \\
& +e_{1}^{2} e_{2}^{2}\left[\eta_{a}{ }^{(2,2)} \widetilde{\alpha}_{a} h^{\alpha}+{ }^{(2,2)} \widetilde{\mu}_{a a} t_{a}^{\alpha}+{ }^{(2,2)} \tilde{\mu}_{a a^{\prime}} t_{a^{\prime}}^{\alpha}\right]
\end{aligned}
$$

with ${ }^{(2,2)} \widetilde{\alpha}_{a}{ }^{(2,2)} \widetilde{\mu}_{a a}$, and ${ }^{(2,2)} \widetilde{\mu}_{a a^{\prime}}$, given by (1.24), (1.28), and (1.25), respectively. So we have calculated the Hamilton-Jacobi momenta, $\tilde{p}_{a}^{\alpha}$, up to terms in $e_{a}^{n} e_{a^{\prime}}^{m}(n+m \leqslant 4)$. Should we need the total 4-momentum, $\widetilde{P}^{\alpha}$, to this order, we only would have to put $\widetilde{P}^{\alpha}=\tilde{p}_{1}^{\alpha}+\tilde{p}_{2}^{\alpha}$.

In the next sections some approximated physical conclusions will be worked out from the expression (1.29). Actually, we will obtain the "radiated" energy, the 3-momentum, and the scattering cross section.

\section{THE "RADIATED" ENERGY AND 3- MOMENTUM}

The approximated Hamilton-Jacobi momenta, $\tilde{p}_{\alpha}^{\alpha}$, given in (1.29) have been defined in such a way that the condition $\lim _{z_{w, z_{a}} \rightarrow \gamma_{\infty} \infty} \tilde{p}_{a}^{\alpha}=\pi_{a}^{\alpha}$, is satisfied, as can be verified directly in (1.29). So, according to whether we take $\gamma=-1$ or $\gamma=+1$, these approximated momenta reduce to the free momenta $\pi_{a}^{\alpha}$, in the infinite past or infinite future, respectively. Nevertheless, in the language of Ref. 1, these momenta, $\tilde{p}_{a}^{\alpha}$, are not conserved, that is, we do not recover the free momenta, $\pi_{a}^{\alpha}$, when taking the limit $z_{a}, z_{a} \rightarrow-\gamma_{\infty}$ as it also can be seen from (1.29). Similar considerations can be made for the total 4-momentum, $\widetilde{P}^{\alpha}=\tilde{p}_{1}^{\alpha}+\tilde{p}_{2}^{\alpha}$. Of course, $\widetilde{P}^{\alpha}$ and 
also $\tilde{p}_{a}^{\alpha}$ are conserved numerically along a given pair of trajectories.

So, let us take the limit $z_{a}, z_{a^{\prime}} \rightarrow-\gamma_{\infty}$ in (1.29). Taking into account (1.28), (1.25), and (1.24) we obtain

$$
\begin{aligned}
& \left.\widetilde{p}_{a}^{\alpha}\right|_{-\gamma \infty} \equiv \lim _{\tilde{z}_{a} \tilde{z}_{a} \rightarrow-\gamma \infty} \tilde{p}_{a}^{\alpha}=\pi_{a}^{\alpha}+e_{1} e_{2} \frac{2 \eta_{a} \gamma \widetilde{k}}{\widetilde{h}^{2} \widetilde{\Lambda}} \widetilde{h}^{\alpha}+e_{1}^{2} e_{2}^{2} \\
& \times\left(-\frac{\eta_{a} \gamma \pi\left(\pi_{a}+\pi_{a^{\prime}}\right)}{2 \widetilde{h}^{\alpha}}-\frac{2 \widetilde{k}^{2}}{\widetilde{\Lambda}^{4} \widetilde{h}^{2}} \widetilde{t}_{a}^{\alpha}+\frac{2 \widetilde{k}^{2}}{\widetilde{\Lambda}^{4} \widetilde{h}^{2}} \widetilde{t}_{a^{\prime}}^{\alpha}\right),
\end{aligned}
$$

where we have restored the symbol $\sim$ because this expression, as compared with (1.29), is a relatively short one.

By making the substitutions ${ }^{1} \pi_{a}^{\alpha} \rightarrow m_{a} u_{a}^{\alpha}$, we obtain $\left.p_{a}^{\alpha}\right|_{-\gamma \infty}$. This is analogous to (2.1), now refering to the original dynamical system. That is,

$$
\begin{aligned}
p_{a}^{\alpha} \mid-\gamma \infty & \\
\equiv & \lim _{z_{i, z_{u}} \rightarrow-\gamma \infty} p_{a}^{\alpha}=m_{a} u_{a}^{\alpha}+e_{1} e_{2} \frac{2 \eta_{a} \gamma k}{h^{2} \Lambda} h^{\alpha}+e_{1}^{2} e_{2}^{2} \\
& \times\left(-\frac{\eta_{a} \gamma \pi\left(m_{1}+m_{2}\right)}{2 m_{1} m_{2} \Lambda h^{3}} h^{\alpha}-\frac{2 k^{2}}{m_{a} \Lambda^{4} h^{2}} t_{a}^{\alpha}\right. \\
& \left.+\frac{2 k^{2}}{m_{a^{\prime}} \Lambda^{4} h^{2}} t_{a^{\prime}}^{\alpha}\right) .
\end{aligned}
$$

Here $k, A, \cdots$ are the quantities of the original system which were given in (I.3.3), (I.3.5), and (I.3.8).

From (2.2) and the total 4-momentum, $P^{\alpha}=p_{1}^{\alpha}+p_{2}^{\alpha}$, we get in our approximation

$$
\left.P^{\alpha}\right|_{-\gamma_{\infty}} \equiv \lim _{z_{i, j} z_{i,} \rightarrow-\gamma_{\infty}} P^{\alpha}=m_{1} u_{1}^{\alpha}+m_{2} u_{2}^{\alpha}
$$

Then the "radiated" 4-momentum up to order $e_{1}^{a} e_{2}^{b}$ with $a+b<6$ is zero. But the intrinsic angular momenta radiated by the two charges is different from zero to order $e_{a} e_{a^{\prime}}^{3}$ (I.5.27) due to the presence of the Dirac term.

We do not know presently if they are "radiation" due to the retarded potential term (I.2.1), because we have not computed the intrinsic angular momentum to order $e_{1}^{2} e_{2}^{2}$. But, if this contribution exists, it would not cancel the $e_{a} e_{a}^{3}$ term (except perhaps for equal charges).

Let us note that the first contribution to radiation comes from the intrinsic angular momentum. Up to order [SI:e:a:1][SI:e:b:2], $a+b=4$, energy and momentum are conserved, but not the intrinsic angular momentum which is tranferred by the system to the universe (from the point of view of the Wheeler-Feynman absorber's theory).

\section{SCATTERING CROSS SECTIONS}

Let us consider (2.2) for $\gamma=1$. Then since the Hamilton-Jacobi momenta, $p_{a}^{\alpha}$ are numerically conserved and since for $\gamma=1$ we have $\lim _{z_{a} z_{a} \rightarrow \infty} p_{a}^{\alpha}=m_{a} u_{a}^{\alpha}$, we can establish the following equation,

$$
\begin{aligned}
& m_{a} u_{a}^{\alpha}+e_{1} e_{2} \frac{2 \eta_{a} k}{\Lambda h^{2}} h^{\alpha}+e_{1}^{2} e_{2}^{2}\left(-\frac{\eta_{a} \pi\left(m_{1}+m_{2}\right)}{2 m_{1} m_{2} \Lambda h^{3}} h^{\alpha}\right. \\
& \left.\quad-\frac{2 k^{2}}{m_{a} \Lambda^{4} h^{2}} t_{a}^{\alpha}+\frac{2 k^{2}}{m_{a^{\prime}} \Lambda^{4} h^{2}} t_{a^{\prime}}^{\alpha}\right) \\
& =m_{a} u_{a F}^{\alpha},
\end{aligned}
$$

where symbols on the left side refer to the initial state and those on the right side to the final state.

From (3.1) and in the laboratory frame, $\mathbf{v}_{a^{\prime}}=0$, we obtain the 3-vector equation

$$
\begin{aligned}
& m_{a} \gamma_{a} \mathbf{v}_{a}+e_{1} e_{2} \frac{2 \eta_{a}}{v_{a} \mathbf{h}^{2}} \mathbf{h}+e_{1}^{2} e_{2}^{2} \\
& \quad \times\left(-\frac{\eta_{a} \pi\left(m_{1}+m_{2}\right)}{2 m_{1} m_{2} \gamma_{a} v_{a}|\mathbf{h}|^{3}} \mathbf{h}-2 \frac{m_{a^{\prime}}+m_{a} \gamma_{a}}{m_{a} m_{a} \gamma_{a} v_{a}^{4} \mathbf{h}^{2}} \mathbf{v}_{a}\right) \\
& =m_{a} \gamma_{a F} \mathbf{v}_{a F} .
\end{aligned}
$$

From the identity $h^{\alpha} u_{a \alpha}=0$, we have $\mathbf{h} \cdot \mathbf{v}_{a}=0$ for $x_{1}^{0}=x_{2}^{0}$. Then Eq. (3.2) is equivalent to the two equations

$m_{a} \gamma_{a} v_{a}-2 e_{1}^{2} e_{2}^{2} \frac{m_{a^{\prime}}+m_{a} \gamma_{a}}{m_{a} m_{a} \gamma_{a} v_{a}^{3} \mathbf{h}^{2}}=m_{a} \gamma_{a F} v_{a F} \cos \theta$,

$\frac{2 e_{1} e_{2}}{v_{a}|\mathbf{h}|}-e_{1}^{2} e_{2}^{2} \frac{\pi\left(m_{1}+m_{2}\right)}{2 m_{1} m_{2} \gamma_{a} v_{a} \mathbf{h}^{2}}=m_{a} \gamma_{a F} v_{a F} \epsilon \sin \theta$,

where $\epsilon$ is the sign of the product $e_{1} e_{2}$ and $\theta$ is the scattering angle between $\mathbf{v}_{a F}$ and $\mathbf{v}_{a}$. From (3.3), (3.4) we have to fourth order

$$
\tan \theta=\frac{2 e_{1} e_{2} \epsilon}{m_{a} \gamma_{a} v_{a}^{2}|\mathbf{h}|}\left(1-\frac{\pi e_{1} e_{2}}{4 m \gamma_{a}|\mathbf{h}|}\right),
$$

where $m$ is the reduced mass: $m \equiv m_{1} m_{2} /\left(m_{1}+m_{2}\right)$. Then

$$
\frac{1}{|\mathbf{h}|}=\frac{2 m \gamma_{a}}{\pi e_{1} e_{2}}\left[1 \pm\left(1-\frac{\epsilon \pi m_{a} v_{a}^{2} \tan \theta}{2 m}\right)^{1 / 2}\right] .
$$

Here we want that $\lim _{\theta \rightarrow 0}|\mathbf{h}|^{-1}=0$ and so only the solution

$$
\frac{1}{|\mathbf{h}|}=\frac{2 m \gamma_{a}}{\pi e_{1} e_{2}}\left[1-\left(1-\frac{\epsilon \pi m_{a} v_{a}^{2} \tan \theta}{2 m}\right)^{1 / 2}\right]
$$

must be retained.

Now the expansion in $e_{1}, e_{2}, \theta$, as a function of $|\mathbf{h}|$ and $\gamma_{a}$, begins with $e_{1} e_{2}$ as it can be seen from (3.5). Taking this into account we can approximate (3.7) to get

$$
|\mathbf{h}|=\frac{2 e_{1} e_{2} \epsilon}{m_{a} \gamma_{a} v_{a}^{2} \sin \theta}-\frac{\pi e_{1} e_{2}}{4 m \gamma_{a}},
$$

where the term $2 e_{1} e_{2} \epsilon / m_{a} \gamma_{a} v_{a}^{2} \sin \theta$ in fact beings with zero order in $e_{1} e_{2}$ because of the $\sin \theta$ appearing in it. Only the other term, $\pi e_{1} e_{2} / 4 m \gamma_{a}$, really behaves as an $e_{1} e_{2}$ term.

From (3.8) we can evaluate the scattering cross section in the LAB frame, $\mathbf{v}_{a^{\prime}}=0$,

$$
d \sigma=\frac{e_{1}^{2} e_{2}^{2}}{4 m_{a}^{2} \gamma_{a}^{2} v_{a}^{2} \sin ^{4}(\theta / 2)}\left(1-\frac{\epsilon \pi m_{a} v_{a}^{2} \sin (\theta / 2)}{4 m}\right) d \Omega,
$$

with $d \Omega$ the differential solid angle. To lowest order, when 
$\left[\epsilon \pi m_{a} v_{a}^{2} \sin (\theta / 2)\right] / 4 m \ll 1$, we obtain

$$
d \sigma=\frac{e_{1}^{2} e_{2}^{2} d \Omega}{4 m_{a}^{2} \gamma_{a}^{2} v_{a}^{2} \sin ^{4}(\theta / 2)}
$$

which is in fact the cross section that would have been obtained if we had neglected terms in $e_{1}^{2} e_{2}^{2}$ in (3.1).

Let us calculate the cross section (3.10) in the center of mass system. This can be easily done by neglecting terms in $e_{1}^{2} e_{2}^{2}$ in (3.1). We find

$$
d \sigma=\frac{e_{1}^{2} e_{2}^{2} k^{2} d \Omega}{4 m_{a}^{2} \gamma_{a}^{2} v_{a}^{2} \Lambda^{2} \sin ^{4}(\theta / 2)} .
$$

It can be seen that this expression agrees with Ref. 3. As it is noted there, (3.11) reduces to Rutherford's formula in the low energy limit and when one of the masses becomes infinite it reduces to Mott's formula for a spinless particle.

Now let us suppose that $m_{a}=m_{a}$, and that after the scattering we cannot distinguish between the two particles. From (3.11) we then find for the scattering cross section

$$
d \sigma=\frac{e_{1}^{2} e_{2}^{2}\left(2 \gamma_{a}^{2}-1\right)^{2}}{m_{a}^{2} v_{a}^{4} \gamma_{a}^{6} \sin ^{4} \theta} .
$$

Akhiezer and Berestetskii, in "Quantum Electrodynamics," p. 838, give (in our notation) to lowest order

$$
d \sigma=\frac{e_{1}^{2} e_{2}^{2}\left(2 \gamma_{a}^{2}-1\right)^{2}}{4 m_{a}^{2} v_{a}^{4} \gamma_{a}^{6}}\left(\frac{2}{\sin ^{2} \theta}-\frac{\gamma_{a}^{2}-1}{2 \gamma_{a}^{2}-1}\right)^{2} d \Omega
$$

for the scattering of two indistinguishable spinless particles in the center of mass system. This formula does not depend on Planck's constant. In (3.13) the expression $\left|\left(\gamma_{a}^{2}-1\right) /\left(2 \gamma_{a}^{2}-1\right)\right|$ is always less than $\frac{1}{2}$. Then we have $2 / \sin ^{2} \theta \gg\left(\gamma_{a}^{2}-1 / 2\right)\left(2 \gamma_{a}^{2}-1\right)$ and (3.13) reduces to (3.12). So, in the appropriate limit, our results agree with those of quantum electrodynamics, at least as far as (3.13) is concerned.

\section{ACKNOWLEDGMENT}

We thank Dr. J. Martin for supplying us with the useful expressions given in Appendices A and B.

\section{APPENDIX A}

The calculation of ${ }^{(1,1)} \theta_{a^{\prime}}\left(\partial^{(1,1)} \tilde{p}_{a}^{\alpha} / \partial \pi^{\alpha^{\prime} \rho}\right)$ becomes easier by systematically using the following relations (J. Martin, private communication):

$$
\begin{aligned}
& N_{a} h^{\alpha}=-z_{a} h^{\alpha}-\eta_{a} \Lambda^{-2} h^{2} t_{a}^{\alpha}, \\
& N_{a} t_{a}^{\alpha}=\eta_{a} \pi_{a}^{2} h^{\alpha}, \\
& N_{a} t_{a^{\prime}}^{\alpha}=\eta_{a^{\prime}} k h^{\alpha}, \\
& N_{a_{a}} z_{a}=\pi_{a}^{2} h^{2} \Lambda^{-2}, N_{a} z_{a^{\prime}}=k h^{2} \Lambda^{-2}, \\
& N_{a} h^{2}=-2 h^{2} z_{a}, N_{a} k=N_{a} \pi_{b}^{2}=0, \\
& Q_{a} h^{\alpha}=0, Q_{a} t_{a}^{\alpha}=-k t_{a}^{\alpha}, Q_{a} t_{a}^{\alpha}=-\pi_{a}^{2} t_{a}^{\alpha}-2 k t_{a^{\prime}}^{\alpha}, \\
& Q_{a} z_{a}=k z_{a}, Q_{a} z_{a^{\prime}}=\pi_{a}^{2} z_{a}, \\
& Q_{a} k=-A^{2}, Q_{a} h^{2}=Q_{a} \pi_{b}^{2}=0,
\end{aligned}
$$

where $N_{a}$ and $P_{a}$ mean the differential operators

$$
N_{a} \equiv \eta_{a} h^{\rho} \frac{\partial}{\partial \pi^{a \rho}}, \quad Q_{a} \equiv t_{a}^{\rho} \frac{\partial}{\partial \pi^{a \rho}} .
$$

\section{APPENDIX B}

$$
\begin{aligned}
& \text { Writing } \\
& { }^{(2,2)} \theta_{a}^{\alpha}=\eta_{a}{ }^{(2,2)} a_{a} h^{\alpha}+{ }^{(2,2)} l_{a a^{\prime}} t_{a}^{\alpha} \text {, }
\end{aligned}
$$

we have for the functions ${ }^{(2,2)} a_{a}$ and ${ }^{(2,2)} l_{a a^{\prime}}$ the following expressions (J. Martin, private communication):

$$
\begin{aligned}
{ }^{(2,2)} a_{a}= & -\pi_{a}^{2} \pi_{a^{4}}^{4} \Lambda^{2} r_{a}^{-2}\left(r_{a}-k z_{a}\right)\left(k r_{a}-\Lambda^{2} z_{a}\right)^{-3} \\
& +\pi_{a}^{2} \pi_{a^{2}}^{2}\left(3 k^{2} h^{2}-\pi_{a}^{2} r_{a}^{2}\right) r_{a}^{-5} \\
& \times\left\{\left(k h^{-2} \pi_{a}^{-2} z_{a^{\prime}} z_{a^{\prime}}+\pi_{a^{\prime}}^{2} \Lambda^{-2}\right) r_{a^{\prime}}^{-1}\right. \\
& -\left[k h^{-2}\left(\pi_{a}^{2} \pi_{a^{\prime}}^{2}\right)^{-1} z_{a}\left(k z_{a}-r_{a}\right)+\pi_{a^{\prime}}^{2} \Lambda^{-2}\right] \\
& \left.\times \pi_{a^{\prime}}^{2}\left(k r_{a}-\Lambda^{2} z_{a}\right)^{-1}\right\} \\
& +3 \pi_{a}^{2} \pi_{a^{2}}^{2} k \Lambda^{2} z_{a} r_{a}^{-5}\left(-\Lambda^{-2}\left(k z_{a}-\pi_{a^{\prime}}^{2} z_{a^{\prime}}\right) r_{a^{\prime}}\right. \\
& +\pi_{a^{\prime}}^{2} \Lambda^{-2} r_{a}\left(k r_{a}-\Lambda^{2} z_{a}\right)^{-1}+\pi_{a^{2}}^{2} \Lambda^{-3} \log \frac{\Lambda z_{a}+r_{a}}{\Lambda z_{a^{\prime}}+r_{a^{\prime}}} \\
& \left.+\pi_{a^{2}}^{2} \Lambda^{-3} \log \frac{k-\Lambda}{\pi_{a^{\prime}}^{2}}\right),
\end{aligned}
$$

$$
\begin{aligned}
{ }^{(2.2)} l_{a a^{\prime}}= & \pi_{a}^{2} \pi_{a^{\prime}}^{6} h^{2} r_{a}^{-2}\left(k r_{a}-\Lambda^{2} z_{a}\right)^{-3}-3 \pi_{a}^{2} \pi_{a^{2}}^{2} k h^{2} z_{a} r_{a}^{-5} \\
& \times\left\{\left[k h^{-2} \pi_{a^{\prime}}^{-2} z_{a^{\prime} a^{\prime}}+\pi_{a^{2}}^{2} \Lambda^{-2}\right] r_{a^{\prime}}^{-1}\right. \\
& -\left[k h^{-2}\left(\pi_{a}^{2} \pi_{a^{\prime}}^{2}\right)^{-1} z_{a}\left(k z_{a}-r_{a}\right)\right. \\
& \left.\left.+\pi_{a^{\prime}}^{2} \Lambda^{-2}\right] \pi_{a^{\prime}}^{2}\left(k r_{a}-\Lambda^{2} z_{a}\right)^{-1}\right\} \\
& -\pi_{a}^{2} \pi_{a^{\prime}}^{2}\left(3 \Lambda^{2} z_{a}^{2}-r_{a}^{2}\right) r_{a}^{-5} \\
& \times\left\{-\Lambda^{-2}\left(k z_{a}-\pi_{a^{\prime}}^{2} z_{a^{\prime}}\right) r_{a^{\prime}}^{-1}\right. \\
& +\Lambda^{-2} \pi_{a^{\prime}}^{2} r_{a}\left(k r_{a}-\Lambda^{2} z_{a}\right)^{-1} \\
& \left.+\pi_{a^{\prime}}^{2} \Lambda^{-3} \log \frac{\Lambda z_{a}+r_{a}}{\Lambda z_{a^{\prime}}+r_{a^{\prime}}}+\pi_{a^{\prime}}^{2} \Lambda^{-3} \log \frac{k-\Lambda}{\pi_{a^{\prime}}^{2}}\right\} .
\end{aligned}
$$

\footnotetext{
'L. Bel and J. Martin, Ann. Inst. H. Poincaré 22, 173 (1975).

${ }^{2}$ L. Bel, "Journées relativistes de Toulouse," Université de Toulouse (1974)

L. Bel, Contribution to Differential Geometry and Relativity, edited by Cohen and Flato (Reidel, Dordrecht, Holland, 1976).

${ }^{4}$ A. Salas and J.M. Sánchez, Nuovo Cimento B 20, 209 (1974).
} 